\title{
STORAGE IMPULSIVE PROCESSES ON INCREASING TIME INTERVALS
}

UDC 519.21

\author{
V. S. KOROLIUK, R. MANCA, AND G. D'AMICO
}

\begin{abstract}
A storage impulsive process $S(t)$ is a sum of (jointly independent) random variables defined on the embedded Markov chain of a homogeneous Markov process.

A storage impulsive process is considered in the scheme of series on increasing time intervals $t / \varepsilon$, with a small parameter $\varepsilon \rightarrow 0, \varepsilon>0$. A storage impulsive process is investigated in the average and diffusion approximation scheme. The large deviation problem is considered under a corresponding scaling with an asymptotically small diffusion.
\end{abstract}

\section{INTRODUCTION}

A storage impulsive process $S(t)$ is a sum of (jointly independent) random variables defined on the embedded Markov chain of a homogeneous Markov process

$$
S(t)=u+\sum_{n=1}^{\nu(t)} \alpha_{n}\left(x_{n}\right), \quad t \geq 0, u \in \mathbb{R}^{d} .
$$

The time homogeneous Markov process $x(t), t \geq 0$, is defined on a standard phase space $(E, \mathcal{E})$ by the generator

$$
Q \varphi(x)=q(x) \int_{E} P(x, d y)[\varphi(y)-\varphi(x)], \quad x \in E,
$$

for a real valued test function $\varphi(x), x \in E$, with a bounded sup-norm:

$$
\|\varphi(x)\|:=\sup _{x \in E}|\varphi(x)| .
$$

The embedded Markov chain $x_{n}, n \geq 0$, is defined by

$$
x_{n}:=x\left(\tau_{n}\right), \quad n \geq 0,
$$

where the renewal moments of jumps are given by

$$
\tau_{n+1}=\tau_{n}+\theta_{n+1}, \quad n \geq 0, \tau_{0}=0,
$$

and the sojourn times $\theta_{n+1}, n \geq 0$, are such that

$$
\mathrm{P}\left(\theta_{n+1} \geq t \mid x_{n}=x\right)=e^{-q(x) t}=: \mathrm{P}\left(\theta_{x} \geq t\right) .
$$

The stochastic kernel $P(x, B), x \in E, B \in \mathcal{E}$, defines the transition probabilities of the embedded Markov chain

$$
P(x, B)=\mathcal{P}\left\{x_{n+1} \in B \mid x_{n}=x\right\} .
$$

2010 Mathematics Subject Classification. Primary 60J45; Secondary 60K05.

Key words and phrases. Storage impulsive process; average, diffusion approximation; large deviation problem. 
The counting process is defined by

$$
\nu(t):=\max \left\{n>0: \tau_{n} \leq t\right\}, \quad t \geq 0 .
$$

The random variables in (1) have the distribution functions

$$
\Phi_{x}(d v)=P\left\{\alpha_{n}(x) \in d v\right\}:=\mathrm{P}\left\{\alpha_{n}\left(x_{n}\right) \in d v \mid x_{n}=x\right\}, \quad x \in E .
$$

A storage impulsive process may be considered as a random evolution process 1 , Chapter 2]. The switching Markov process $x(t), t \geq 0$, describes a random environment.

A1: The main assumption is the uniform ergodicity of the Markov process $x(t), t \geq 0$, with the stationary distribution $\pi(B), B \in \mathcal{E}$, satisfying the equation

$$
\pi(d x) q(x)=q \rho(d x), \quad q=\int_{E} \pi(d x) q(x) .
$$

The stationary distribution $\rho(B), B \in \mathcal{E}$, of the embedded Markov chain $x_{n}, n \geq 0$, satisfies the equation

$$
\rho(B)=\int_{E} \rho(d x) P(x, B), \quad B \in \mathcal{E}, \rho(E)=1 .
$$

If the main assumption $\mathbf{A} \mathbf{1}$ holds, then the potential operator $R_{0}$ may be given by a solution of the equation [1, Chapter 2]

$$
Q R_{0}=R_{0} Q=\Pi-I, \quad \Pi \varphi(x):=\int_{E} \pi(d x) \varphi(x)
$$

\section{A STORAGE IMPULSIVE PROCESS ON INCREASING TIME INTERVALS IN AN AVERAGE SCHEME}

A storage impulsive process on increasing time intervals in an average scheme is considered in the scheme of series with a small parameter $\varepsilon \rightarrow 0, \varepsilon>0$, in the following scaling:

$$
S^{\varepsilon}(t)=u+\varepsilon \sum_{n=1}^{\nu(t / \varepsilon)} \alpha_{n}\left(x_{n}\right), \quad t \geq 0, \varepsilon>0, u \in \mathbb{R}^{d} .
$$

The random evolution approach [1, Chapter 3,5] is an effective method of asymptotic analysis (2) as $\varepsilon \rightarrow 0$.

Proposition 2.1. Storage impulsive process (2) in the average scheme convergences weakly,

$$
S^{\varepsilon}(t) \Rightarrow S^{0}(t)=u+\widehat{a}_{0} t, \quad \varepsilon \rightarrow 0,
$$

where the average velocity is such that

$$
\widehat{a}_{0}=q \widehat{a}, \quad \widehat{a}=\int_{E} \rho(d x) a(x), \quad a(x)=\int_{\mathbb{R}^{d}} v \Phi_{x}(d v) .
$$

Proof of Proposition 2.1 is based on the random evolution approach [1, Chapter 3] by using a solution of the singular perturbation problem [1, Chapter 5].

Remark 2.1. For simplicity and without loss of generality, the proof is given for a storage impulsive process defined on the real line $\mathbb{R}, d=1$.

According to the definition of a random evolution [1, Chapter 2] we consider a two component Markov process,

$$
S^{\varepsilon}(t), x^{\varepsilon}(t):=x(t / \varepsilon), \quad t \geq 0 .
$$


Lemma 2.1. Markov process (5) is characterized by the generator

$$
L^{\varepsilon} \varphi(u, x)=\varepsilon^{-1} q(x) \int_{E} P(x, d y) \int_{\mathbb{R}^{d}} \Phi_{y}(d v)[\varphi(u+\varepsilon v, y)-\varphi(u, x)] .
$$

Lemma 2.1 is a direct consequence of the definition of the generator [1, Chapter 3].

Remark 2.2. Generator (6) may be rewritten as

$$
L^{\varepsilon} \varphi(u, x)=\varepsilon^{-1}\left[Q+Q_{0} \Phi_{x}^{\varepsilon}\right] \varphi(u, x),
$$

where, by definition,

$$
\begin{gathered}
Q_{0} \varphi(x):=q(x) \int_{E} P(x, d y) \varphi(y), \\
\Phi_{x}^{\varepsilon} \varphi(u):=\int_{\mathbb{R}^{d}} \Phi_{x}(d v)[\varphi(u+\varepsilon v)-\varphi(u)] .
\end{gathered}
$$

On a test function $\varphi(u)$ being smooth enough,

$$
\Phi_{x}^{\varepsilon} \varphi(u)=\varepsilon\left[a(x) \varphi^{\prime}(u)+\delta^{\varepsilon}(x) \varphi(u)\right]
$$

with the negligible term

$$
\left\|\delta^{\varepsilon}(x) \varphi(u)\right\| \rightarrow 0, \quad \varepsilon \rightarrow 0, \quad \varphi(u) \in C^{2}(\mathbb{R}) .
$$

Lemma 2.2. Generator (7) admits the following asymptotic expansion:

$$
L^{\varepsilon} \varphi(u, x)=\left[\varepsilon^{-1} Q+Q_{0} \mathbb{A}(x)+\delta^{\varepsilon}(x)\right] \varphi(u, x),
$$

where

$$
\mathbb{A}(x) \varphi(u):=a(x) \varphi^{\prime}(u),
$$

and the negligible term is such that

$$
\sup _{x \in E}\left\|\delta^{\varepsilon}(x) \varphi(u, x)\right\| \rightarrow 0, \quad \varepsilon \rightarrow 0, \quad \varphi(u, \cdot) \in C^{2}(\mathbb{R}) .
$$

Then a solution of the singular perturbation problem [1, Chapter 5] may be used for the truncated operator

$$
L_{0}^{\varepsilon} \varphi(u, x):=\left[\varepsilon^{-1} Q+Q_{0} \mathbb{A}(x)\right] \varphi(u, x) .
$$

Lemma 2.3. Truncated operator (8) on a perturbed test function

$$
\varphi^{\varepsilon}(u, x)=\varphi(u)+\varepsilon \varphi_{1}(u, x),
$$

admits the following asymptotic representation [1, Proposition 5.1]:

$$
L_{0}^{\varepsilon} \varphi^{\varepsilon}(u, x)=\widehat{a}_{0} \varphi^{\prime}(u)+\delta^{\varepsilon}(x) \varphi(u) .
$$

The negligible term may be written in an explicit form:

$$
\begin{gathered}
\delta^{\varepsilon}(x) \varphi(u)=\varepsilon Q_{0} \mathbb{A}(x) R_{0} \widehat{\mathbb{A}}(x) \varphi(u), \\
\widehat{\mathbb{A}}(x):=\widehat{\mathbb{A}}_{0}-Q_{0} \mathbb{A}(x), \quad \widehat{\mathbb{A}}_{0}:=\Pi Q_{0} \mathbb{A}(x) \Pi .
\end{gathered}
$$

Conclusion 2.1. Generator (6) of random evolution (5) admits the asymptotic representation

$$
L^{\varepsilon} \varphi^{\varepsilon}(u, x)=\widehat{a}_{0} \varphi^{\prime}(u)+\delta^{\varepsilon}(x) \varphi(u)
$$

with the negligible term $\delta^{\varepsilon}(x) \varphi(u)$.

Representation (9) implies weak convergence (3)-(4) [1, Chapter 6] since the limit operator,

$$
L^{0} \varphi(u):=\widehat{a}_{0} \varphi^{\prime}(u), \quad \varphi(u) \in C^{1}(\mathbb{R}),
$$


defines the evolution

$$
S^{0}(t)=u+\widehat{a}_{0} t, \quad t \geq 0, S^{0}(0)=u .
$$

Remark 2.3. Limit operator (10) in the Euclidean space $\mathbb{R}^{d}$ has the following representation:

$$
\begin{gathered}
\widehat{a}_{0} \varphi^{\prime}(u):=\sum_{k=1}^{d} \widehat{a}_{k}^{0} \varphi_{k}^{\prime}(u), \quad \varphi_{k}^{\prime}(u):=\partial \varphi(u) / \partial u_{k}, \\
\widehat{a}_{k}^{0}=q \widehat{a}_{k}, \quad \widehat{a}_{k}=\int_{E} \rho(d x) a_{k}(x), \quad a_{k}(x)=\int_{\mathbb{R}} v_{k} \Phi_{x}(d v) .
\end{gathered}
$$

\section{A STORAGE IMPULSIVE PROCESS IN A SCHEME OF DIFFUSION APPROXIMATION}

It is well known that the diffusion approximation of stochastic systems may be realized under some additional Balance Condition.

We consider two different balance conditions for a storage impulsive process, namely the total and local ones.

3.1. A storage impulsive process under the total balance condition. A storage impulsive process in the scheme of series with the parameter $\varepsilon \rightarrow 0, \varepsilon>0$, in the diffusion approximation scheme under the Total Balance Condition,

$$
a(x)=\int_{\mathbb{R}^{d}} v \Phi_{x}(d v) \equiv 0,
$$

is considered in the following scaling:

$$
S^{\varepsilon}(t)=u+\varepsilon \sum_{n=1}^{\nu\left(t / \varepsilon^{2}\right)} \alpha_{n}\left(x_{n}\right), \quad t \geq 0, \varepsilon>0 .
$$

Proposition 3.1. If total balance condition (11) holds, then

$$
S^{\varepsilon}(t) \Rightarrow W_{\sigma}(t), \quad \varepsilon \rightarrow 0 .
$$

The limit Brownian motion $W_{\sigma}(t), t \geq 0$, is defined by the variance matrix

$$
\begin{gathered}
\widehat{C}=\sigma^{*} \sigma=q \widehat{B}, \\
\widehat{B}=\int_{E} \rho(d x) B(x), \quad B(x)=\int_{\mathbb{R}^{d}} v^{*} v \Phi_{x}(d v) .
\end{gathered}
$$

Proof of Proposition 3.1. As in Section 2, we start by characterizing the coupled Markov process.

Lemma 3.1. The Markov process

$$
S^{\varepsilon}(t), x^{\varepsilon}(t):=x\left(t / \varepsilon^{2}\right), \quad t \geq 0,
$$

is characterized by the generator

$$
L^{\varepsilon} \varphi(u, x)=\varepsilon^{-2} q(x) \int_{E} P(x, d y) \int_{\mathbb{R}^{d}} \Phi_{x}(d v)[\varphi(u+\varepsilon v, y)-\varphi(u, x)] .
$$

Generator (12) can be rewritten as

$$
L^{\varepsilon} \varphi(u, x)=\varepsilon^{-2}\left[Q+Q_{0} \Phi_{x}^{\varepsilon}\right] \varphi(u, x),
$$

where

$$
\Phi_{x}^{\varepsilon} \varphi(u):=\int_{\mathbb{R}^{d}} \Phi_{x}(d v)[\varphi(u+\varepsilon v)-\varphi(u)]=\varepsilon^{2}\left[\frac{1}{2} B(x) \varphi^{\prime \prime}(u)+\delta^{\varepsilon}(x) \varphi(u)\right],
$$

with the negligible term $\delta^{\varepsilon}(x) \varphi(u)$. 
Lemma 3.2. Generator (13)-(14) admits the following asymptotic expansion

$$
L^{\varepsilon} \varphi(u, x)=\left[\varepsilon^{-2} Q+Q_{0} \mathbb{B}(x)\right] \varphi(u, x)+\delta^{\varepsilon}(x) \varphi(u)
$$

with the negligible term $\delta^{\varepsilon}(x) \varphi(u)$. Here, by definition,

$$
\mathbb{B}(x) \varphi(u)=\frac{1}{2} B(x) \varphi^{\prime \prime}(u) .
$$

Then the solution of singular perturbation problem [1, Chapter 5] can be used for the truncated operator

$$
\mathbb{L}_{0}^{\varepsilon} \varphi(u, x)=\left[\varepsilon^{-2} Q+Q_{0} \mathbb{B}(x)\right] \varphi(u, x) .
$$

Lemma 3.3. Truncated operator (16) on a perturbed test function

$$
\varphi^{\varepsilon}(u, x)=\varphi(u)+\varepsilon^{2} \varphi_{2}(u, x),
$$

admits the asymptotic representation

$$
L_{0}^{\varepsilon} \varphi^{\varepsilon}(u, x)=\frac{1}{2} \widehat{C} \varphi^{\prime \prime}(u)+\delta^{\varepsilon}(x) \varphi(u) .
$$

Proof. Considering (16) and (17),

$$
\begin{aligned}
L_{0}^{\varepsilon} \varphi^{\varepsilon} & =\left[\varepsilon^{-2} Q+Q_{0} \mathbb{B}(x)\right]\left[\varphi(u)+\varepsilon^{2} \varphi_{2}(u, x)\right] \\
& =\varepsilon^{-2} Q \varphi(u)+\left[Q \varphi_{2}(u, x)+Q_{0} \mathbb{B}(x) \varphi(u)\right]+\delta^{\varepsilon}(x) \varphi(u) .
\end{aligned}
$$

It is obvious that

The equation

$$
Q \varphi(u)=0
$$

$$
Q \varphi_{2}(u, x)+Q_{0} \mathbb{B}(x) \varphi(u)=\widehat{L}_{0} \varphi(u)
$$

can be solved under the solvability condition [1, Chapter 5]:

$$
\widehat{L}_{0} \Pi=\Pi Q_{0} \mathbb{B}(x) \Pi \text {. }
$$

Transforming (15) gives us

Indeed,

$$
\widehat{L}_{0} \varphi(u)=\frac{1}{2} \widehat{C} \varphi^{\prime \prime}(u)
$$

$$
\begin{aligned}
\widehat{L}_{0} \varphi(u) & =\int_{E} \pi(d x) q(x) \int_{E} P(x, d y) \frac{1}{2} B(y) \varphi^{\prime \prime}(u) \\
& =\frac{1}{2} q \int_{E} \rho(d x) B(x) \varphi^{\prime \prime}(u)=\frac{1}{2} q \widehat{B} \varphi^{\prime \prime}(u) .
\end{aligned}
$$

Remark 3.1. The limit generator $\widehat{L}_{0}$ in the Euclidean space $\mathbb{R}^{d}$ is represented as follows:

$$
\begin{gathered}
\widehat{L}_{0} \varphi(u)=\frac{q}{2} \sum_{k, r=1}^{d} B_{k r} \varphi_{k r}^{\prime \prime}(u), \\
\widehat{B}=\left[B_{k r} ; 1 \leq k, r \leq d\right], \quad \varphi_{k r}^{\prime \prime}(u):=\partial^{2} \varphi(u) / \partial u_{k} \partial u_{r}, \\
B_{k r}=\int_{E} \rho(d x) B_{k r}(x), \quad B_{k r}(x)=\int_{\mathbb{R}} v_{k} v_{r} \Phi_{x}(d v) .
\end{gathered}
$$

We complete the proof of Proposition 3.1 by using the asymptotic representation

$$
L^{\varepsilon} \varphi^{\varepsilon}(u, x)=\widehat{L}_{0} \varphi(u)+\delta^{\varepsilon}(x) \varphi(u)
$$

and convergence Theorem 6.3 in [1, Chapter 6]. The negligible term in (18) can be written in an explicit form. 
3.2. A storage impulsive process under the local balance condition. The local balance condition means that the average value of jumps is such that

$$
\widehat{a}:=\int_{E} \rho(d x) a(x) \neq 0 .
$$

A storage impulsive process in the scheme of series under local balance condition (19) with the parameter $\varepsilon \rightarrow 0, \varepsilon>0$, is considered in the following scaling:

$$
S^{\varepsilon}(t)=u+\varepsilon \sum_{n=1}^{\nu\left(t / \varepsilon^{2}\right)} \alpha_{n}\left(x_{n}\right)-q \widehat{a} t / \varepsilon, \quad t \geq 0 .
$$

Proposition 3.2. If local balance condition (19) holds, then

$$
S^{\varepsilon}(t) \Rightarrow W_{\sigma}(t), \quad \varepsilon \rightarrow 0 .
$$

The limit Brownian motion $W_{\sigma}(t), t \geq 0$, is defined by the variance matrix

$$
\begin{gathered}
\widehat{C}=\sigma^{*} \sigma=q \widehat{B}, \quad \widehat{B}=\widehat{B}_{0}+\widehat{B}_{1}, \\
\widehat{B}_{0}=\int_{E} \rho(d x) B_{0}(x), \quad B_{0}(x)=\int_{\mathbb{R}^{d}} v^{*} v \Phi_{x}(d v), \\
\widehat{B}_{1}=\int_{E} \rho(d x) B_{1}(x), \quad B_{1}(x)=2 \widehat{a}^{*}(x) R_{0} \widehat{a}(x), \\
\widehat{a}(x):=a_{0}(x)-q \widehat{a}, \\
a_{0}(x):=q(x) \int_{E} P(x, d y) a(y) .
\end{gathered}
$$

Here the potential operator $R_{0}$ is defined as the solution of the equation

$$
Q R_{0}=R_{0} Q=\Pi-I
$$

(see [1, Chapter 3]).

Proof of Proposition 3.2, As in the previous section, we use the generator of the two component Markov process.

Lemma 3.4. The two component Markov process $S^{\varepsilon}(t), x^{\varepsilon}(t):=x\left(t / \varepsilon^{2}\right), t \geq 0$, is characterized by the generator

(22) $L^{\varepsilon} \varphi(u, x)=\varepsilon^{-2} q(x) \int_{E} P(x, d y) \int_{\mathbb{R}^{d}} \Phi(d v)[\varphi(u+\varepsilon v, y)-\varphi(u, x)]-\varepsilon^{-1} \widehat{a}_{0} \varphi^{\prime}(u, x)$.

This generator can be written as

$$
L^{\varepsilon} \varphi(u, x)=\left[\varepsilon^{-2}\left[Q+Q_{0} \Phi_{x}^{\varepsilon}\right]-\varepsilon^{-1} \widehat{\mathbb{A}}_{0}\right] \varphi(u, x)
$$

with $\widehat{\mathbb{A}}_{0} \varphi(u):=\widehat{a}_{0} \varphi^{\prime}(u)$,

$$
\begin{aligned}
\Phi_{x}^{\varepsilon} \varphi(u) & =\int_{\mathbb{R}^{d}} \Phi_{x}(d v)[\varphi(u+\varepsilon v)-\varphi(u)] \\
& =\varepsilon a(x) \varphi^{\prime}(u)+\varepsilon^{2} \frac{1}{2} B(x) \varphi^{\prime \prime}(u)+\varepsilon^{2} \delta^{\varepsilon}(x) \varphi(u) .
\end{aligned}
$$

Lemma 3.5. Generator (22) admits the following asymptotic expansion:

$$
L^{\varepsilon} \varphi(u, x)=\left[\varepsilon^{-2} Q+\varepsilon^{-1} \widehat{\mathbb{A}}(x)+Q_{0} \mathbb{B}(x)\right] \varphi(u, x)+\delta^{\varepsilon}(x) \varphi(u, x) .
$$


Here

$$
\begin{gathered}
\widehat{\mathbb{A}}(x) \varphi(u)=\widehat{a}(x) \varphi^{\prime}(u), \\
\widehat{a}(x):=a_{0}(x)-\widehat{a}_{0}, \\
a_{0}(x):=q(x) \int_{E} P(x, d y) a(y) .
\end{gathered}
$$

Note that the following balance condition holds:

$$
\Pi \widehat{a}(x)=0 .
$$

Now a solution of the singular perturbation problem [1, Chapter 5] can be used for the truncated operator

$$
L_{0}^{\varepsilon} \varphi(u, x)=\left[\varepsilon^{-2} Q+\varepsilon^{-1} \widehat{\mathbb{A}}(x)+Q_{0} \mathbb{B}(x)\right] \varphi(u, x) .
$$

Lemma 3.6. Truncated operator (26) on a perturbed test function

$$
\varphi^{\varepsilon}(u, x)=\varphi(u)+\varepsilon \varphi_{1}(u, x)+\varepsilon^{2} \varphi_{2}(u, x)
$$

admits the following asymptotic representation:

$$
L_{0}^{\varepsilon} \varphi^{\varepsilon}(u, x)=\frac{1}{2} \widehat{C} \varphi^{\prime \prime}(u)+\delta^{\varepsilon}(x) \varphi(u) .
$$

Proof. Consider

$$
\begin{aligned}
L_{0}^{\varepsilon} \varphi^{\varepsilon}(u, x)= & {\left[\varepsilon^{-2} Q+\varepsilon^{-1} \widehat{\mathbb{A}}(x)+Q_{0} \mathbb{B}(x)\right]\left[\varphi(u)+\varepsilon \varphi_{1}(u, x)+\varepsilon^{2} \varphi_{2}(u, x)\right] } \\
= & \varepsilon^{-2} Q \varphi(u)+\varepsilon^{-1}\left[Q \varphi_{1}+\widehat{\mathbb{A}}(x) \varphi\right]+\left[Q \varphi_{2}+\widehat{\mathbb{A}}(x) \varphi_{1}+Q_{0} \mathbb{B}(x) \varphi\right] \\
& +\delta^{\varepsilon}(x) \varphi(u) .
\end{aligned}
$$

Then

$$
\begin{gathered}
Q \varphi(u)=0, \\
Q \varphi_{1}(u, x)+\widehat{\mathbb{A}}(x) \varphi(u)=0, \\
Q \varphi_{2}(u, x)+\widehat{\mathbb{A}}(x) \varphi_{1}(u, x)+Q_{0} \mathbb{B}(x) \varphi(u)=\widehat{L}_{0} \varphi(u) .
\end{gathered}
$$

The first equation above is obvious. The second equation satisfies the solvability condition (25). Hence

Now the third equation is

$$
\varphi_{1}(u, x)=R_{0} \widehat{\mathbb{A}}(x) \varphi(u) .
$$

$$
Q \varphi_{2}+\left[\widehat{\mathbb{A}}_{0}(x)+Q_{0} \mathbb{B}(x)\right] \varphi(u)=\widehat{L}_{0} \varphi(u),
$$

where

$$
\widehat{\mathbb{A}}_{0}(x) \varphi(u):=\widehat{\mathbb{A}}(x) R_{0} \widehat{\mathbb{A}}(x) \varphi(u) .
$$

The solvability condition for (27) implies

$$
\widehat{L}_{0} \Pi=\Pi\left[\widehat{\mathbb{A}}_{0}(x)+Q_{0} \mathbb{B}(x)\right] \Pi .
$$

Using (28), (24), and (15) we evaluate the limit generator

$$
\widehat{L}_{0} \varphi(u)=\frac{1}{2} \widehat{C} \varphi^{\prime \prime}(u),
$$

where the variance matrix $\widehat{C}$ is given by (21).

Note that (see (24))

$\widehat{\mathbb{A}}_{0}(x) \varphi(u)=\widehat{\mathbb{A}}(x) R_{0} \widehat{\mathbb{A}}(x) \varphi(u)=\widehat{\mathbb{A}}(x) R_{0} \widehat{a}(x) \varphi^{\prime}(u)=\widehat{a}(x) R_{0} \widehat{a}(x) \varphi^{\prime \prime}(u)=\frac{1}{2} B_{1}(x) \varphi^{\prime \prime}(u)$. 
Here

$$
\widehat{a}(x)=a_{0}(x)-\widehat{a}_{0} .
$$

The proof of Lemma 3.6 and Proposition 3.2 is complete.

4. LARGE DEVIATION IN THE SCHEME OF AN ASYMPTOTICALLY SMALL DIFFUSION

A storage impulsive process in the scheme of an asymptotically small diffusion is considered under two different balance conditions, namely total and local ones.

4.1. A storage impulsive process under the total balance condition. The total balance condition means that the mean values of jumps of a storage impulsive process equal, totally, zero:

$$
a(x)=\int_{\mathbb{R}^{d}} v \Phi_{x}(d v) \equiv 0 .
$$

A storage impulsive process in the scheme of an asymptotically small diffusion is considered in the following scaling [3]:

$$
S^{\varepsilon}(t)=u+\varepsilon^{2} \sum_{n=1}^{\nu\left(t / \varepsilon^{3}\right)} \alpha_{n}\left(x_{n}\right), \quad t \geq 0, \varepsilon>0, u \in \mathbb{R}^{d} .
$$

The coupled Markov process

$$
S^{\varepsilon}(t), x^{\varepsilon}(t):=x\left(t / \varepsilon^{3}\right), \quad t \geq 0,
$$

is defined by the generator

$$
L^{\varepsilon} \varphi(u, x)=\varepsilon^{-3} q(x) \int_{E} P(x, d y) \int_{\mathbb{R}^{d}} \Phi_{y}(d v)\left[\varphi\left(u+\varepsilon^{2} v, y\right)-\varphi(u, x)\right],
$$

which can be rewritten as

$$
L^{\varepsilon} \varphi(u, x)=\varepsilon^{-3}\left[Q+Q_{0} \Phi_{x}^{\varepsilon}\right] \varphi(u, x),
$$

where, by definition,

$$
\Phi_{x}^{\varepsilon} \varphi(u):=\int_{\mathbb{R}^{d}} \Phi_{x}(d v)\left[\varphi\left(u+\varepsilon^{2} v\right)-\varphi(u)\right]=\varepsilon^{4}\left[\mathbb{B}(x) \varphi(u)+\delta^{\varepsilon}(x) \varphi(u)\right] .
$$

Here

$$
\mathbb{B}(x) \varphi(u):=\frac{1}{2} B(x) \varphi^{\prime \prime}(u) .
$$

Hence generator (31) admits the asymptotic expansion

$$
\begin{gathered}
L^{\varepsilon} \varphi(u, x)=L_{0}^{\varepsilon} \varphi(u, x)+\delta^{\varepsilon}(x) \varphi(u, x), \\
L_{0}^{\varepsilon} \varphi(u, x)=\left[\varepsilon^{-3} Q+\varepsilon Q_{0} \mathbb{B}(x)\right] \varphi(u, x) .
\end{gathered}
$$

Truncated operator (32) on a perturbed test function,

$$
\varphi^{\varepsilon}(u, x)=\varphi(u)+\varepsilon^{4} \varphi_{1}(u, x),
$$

admits the asymptotic representation

$$
L_{0}^{\varepsilon} \varphi^{\varepsilon}(u, x)=\varepsilon\left[Q \varphi_{1}+Q_{0} \mathbb{B}(x) \varphi(u)\right]+\delta^{\varepsilon}(x) \varphi(u) .
$$

Representations (32) and (33) give

where the main part

$$
L^{\varepsilon} \varphi^{\varepsilon}(u, x)=\varepsilon\left[\widehat{\mathbb{C}} \varphi(u)+\delta^{\varepsilon}(x) \varphi(u, x)\right],
$$

is the generator of a small diffusion.

$$
\varepsilon \widehat{\mathbb{C}} \varphi(u)=\varepsilon \frac{1}{2} \widehat{C} \varphi^{\prime \prime}(u)
$$


4.2. Large deviation for a storage impulsive process under the total balance condition. We investigate the large deviation problem for a storage impulsive process by using the asymptotic analysis of the exponential generator of the large deviation

$$
H^{\varepsilon} \varphi(u, x)=e^{-\varphi / \varepsilon} \varepsilon L^{\varepsilon} e^{\varphi / \varepsilon}
$$

(see [2, Part I]).

Proposition 4.1. The large deviation of a storage impulsive process (30) under the total balance condition (29) is realized by the exponential generator of a small diffusion,

$$
\begin{gathered}
H \varphi(u)=\frac{1}{2} \widehat{C}\left[\varphi^{\prime}(u)\right]^{2}, \\
\widehat{C}=q \int_{E} \rho(d x) B(x), \quad B(x)=\int_{\mathbb{R}^{d}} v^{*} v \Phi_{x}(d v) .
\end{gathered}
$$

Proof of Proposition 4.1, The proof is based on the following auxiliary result.

Lemma 4.1. Exponential generator (34) on a perturbed test function,

$$
\varphi^{\varepsilon}(u, x)=\varphi(u)+\varepsilon \ln \left[1+\varepsilon^{2} \varphi_{1}(u, x)\right]
$$

admits the asymptotic representation

$$
H^{\varepsilon} \varphi^{\varepsilon}(u, x)=Q \varphi_{1}+\frac{1}{2} Q_{0} B(x)\left[\varphi^{\prime}(u)\right]^{2}+h^{\varepsilon}(x) \varphi(u)
$$

with the negligible term

$$
\left\|h^{\varepsilon}(x) \varphi(u)\right\| \rightarrow 0, \quad \varepsilon \rightarrow 0, \quad \varphi(u) \in C^{3}(\mathbb{R}) .
$$

Proof of Lemma 4.1. It is clear that

$$
\begin{aligned}
H^{\varepsilon} \varphi^{\varepsilon} & =e^{-\varphi / \varepsilon}\left[1+\varepsilon^{2} \varphi_{1}\right]^{-1} \varepsilon L^{\varepsilon}\left[1+\varepsilon^{2} \varphi_{1}\right] e^{\varphi / \varepsilon} \\
& =e^{-\varphi / \varepsilon}\left[1-\varepsilon^{2} \varphi_{1}\right] \varepsilon L_{0}^{\varepsilon}\left[1+\varepsilon^{2} \varphi_{1}\right] e^{\varphi / \varepsilon}+h^{\varepsilon}(x) \varphi(u) \\
& =e^{-\varphi / \varepsilon}\left[1-\varepsilon^{2} \varphi_{1}\right] \varepsilon^{-2} Q\left[1+\varepsilon^{2} \varphi_{1}\right] e^{\varphi / \varepsilon}+e^{-\varphi / \varepsilon} \varepsilon^{-2} Q_{0} \Phi_{x}^{\varepsilon} e^{\varphi / \varepsilon}+h^{\varepsilon}(x) \varphi(u) \\
& =Q \varphi_{1}+\frac{1}{2} Q_{0} B(x)\left[\varphi^{\prime}(u)\right]^{2}+h^{\varepsilon}(x) \varphi(u) .
\end{aligned}
$$

Turning back to the proof of Proposition 4.1, the solution of the singular perturbation problem [1, Chapter 5] gives

$$
H^{\varepsilon} \varphi^{\varepsilon}(u, x)=H \varphi(u)+h^{\varepsilon}(x) \varphi(u) .
$$

The asymptotic representation (36) completes the proof of Proposition 4.1 .

Remark 4.1. The exponential generator of small diffusion (35) in the Euclidean space $\mathbb{R}^{d}$, $d \geq 2$, is represented as

$$
H \varphi(u)=\frac{1}{2} \varphi^{\prime *}(u) \widehat{C} \varphi^{\prime}(u),
$$

where $\varphi^{\prime *}(u)=\left(\varphi_{k}^{\prime}(u), 1 \leq k \leq d\right)$ is a vector-row, $\varphi^{\prime}(u)=\left(\varphi_{k}^{\prime}(u), 1 \leq k \leq d\right)$ is a vector-column, and $\widehat{C}=\left[\widehat{C}_{k r ; 1 \leq k, r \leq d}\right]$ is the variance matrix. 
4.3. Large deviation for a storage impulsive process under the local balance condition. The Local Balance Condition means that the average value of jumps is not equal to zero:

$$
\widehat{a}:=\int_{E} \rho(d x) a(x) \neq 0 .
$$

A storage impulsive process under local balance condition (37) is considered in the following scaling:

$$
S^{\varepsilon}(t)=u+\varepsilon^{2} \sum_{n=1}^{\nu\left(t / \varepsilon^{3}\right)} \alpha_{n}\left(x_{n}\right)-\widehat{a}_{0} t / \varepsilon .
$$

Lemma 4.2. The coupled Markov process $S^{\varepsilon}(t), x^{\varepsilon}(t):=x\left(t / \varepsilon^{3}\right), t \geq 0$, is determined by the generator ( $c f .(22)$ )

$$
L^{\varepsilon} \varphi(u, x)=\varepsilon^{-3} q(x) \int_{E} P(x, d y) \int_{\mathbb{R}^{d}} \Phi_{y}(d v)\left[\varphi\left(u+\varepsilon^{2} v, y\right)-\varphi(u, x)\right]-\varepsilon^{-1} \widehat{a}_{0} \varphi_{u}^{\prime}(u, x) .
$$

Or, in a different form,

$$
\begin{aligned}
L^{\varepsilon} \varphi(u, x) & =\left[\varepsilon^{-3}\left[Q+Q_{0} \Phi_{x}^{\varepsilon}\right]-\varepsilon^{-1} \widehat{\mathbb{A}}_{0}\right] \varphi(u, x), \\
\Phi_{x}^{\varepsilon} \varphi(u) & =\int_{\mathbb{R}^{d}} \Phi_{x}(d v)\left[\varphi\left(u+\varepsilon^{2} v\right)-\varphi(u)\right] .
\end{aligned}
$$

Proposition 4.2. The large deviation for a storage impulsive process (38) under local balance condition (37) is realized by the exponential generator of a small diffusion

$$
\begin{gathered}
H \varphi(u)=\frac{1}{2} \widehat{C}\left[\varphi^{\prime}(u)\right]^{2}, \\
\widehat{C}=q\left[\widehat{B}_{1}+\widehat{B}_{2}\right], \\
\widehat{B}_{k}=\int_{E} \rho(d x) B_{k}(x), \quad k=1,2, \\
B_{1}(x)=\int_{\mathbb{R}^{d}} v^{*} v \Phi_{x}(d v), \quad B_{2}(x)=2 \widehat{a}(x) R_{0} \widehat{a}(x), \\
\widehat{a}(x)=a_{0}(x)-\widehat{a}_{0}, \quad a_{0}(x):=q(x) \int_{E} P(x, d y) a(x) .
\end{gathered}
$$

The exponential generator of large deviation (39)-(40) contains two components. One of them is the variance matrix of the second moment of jumps. The second component $\widehat{B}_{2}$ is defined by the fluctuation of the first moment of jumps.

Proof of Proposition 4.2. The proof is based on the following auxiliary result.

Lemma 4.3. Exponential generator (34) under local balance condition (37) on the perturbed test function

$$
\varphi^{\varepsilon}(u, x)=\varphi(u)+\varepsilon \ln \left[1+\varepsilon \varphi_{1}(u, x)+\varepsilon^{2} \varphi_{2}(u, x)\right]
$$

admits the asymptotic representation

$$
\begin{aligned}
H^{\varepsilon} \varphi^{\varepsilon}(u, x)= & \varepsilon^{-1}\left[Q \varphi_{1}+\widetilde{\mathbb{A}}(x) \varphi(u)\right] \\
& +\left[Q \varphi_{2}-\varphi_{1} Q \varphi_{1}+\frac{1}{2} Q_{0} B(x)\left[\varphi^{\prime}(u)\right]^{2}\right]+h^{\varepsilon}(x) \varphi(u)
\end{aligned}
$$

with the negligible term

$$
\left\|h^{\varepsilon}(x) \varphi(u)\right\| \rightarrow 0, \quad \varepsilon \rightarrow 0, \quad \varphi(u) \in C^{3}(\mathbb{R}) .
$$


Proof. Note the following asymptotic representations:

$$
\begin{gathered}
H_{Q}^{\varepsilon} \varphi^{\varepsilon}(u, x):=e^{-\varphi^{\varepsilon} / \varepsilon} \varepsilon^{-2} Q e^{\varphi^{\varepsilon} / \varepsilon}=\varepsilon^{-1} Q \varphi_{1}+\left[Q \varphi_{2}-\varphi_{1} Q \varphi_{1}\right]+h_{q}^{\varepsilon}(x) \varphi(u), \\
H_{\varphi}^{\varepsilon} \varphi^{\varepsilon}(u, x):=e^{-\varphi^{\varepsilon} / \varepsilon} \varepsilon^{-2} Q_{0} \Phi_{x}^{\varepsilon} e^{\varphi^{\varepsilon} / \varepsilon}=\varepsilon^{-1} Q_{0} \mathbb{A}(x) \varphi(u)+Q_{0} \mathbb{A}(x) \varphi_{1}(u, x)+h_{\varphi}^{\varepsilon}(x) \varphi(u), \\
H_{a}^{\varepsilon} \varphi^{\varepsilon}(u, x):=e^{-\varphi^{\varepsilon} / \varepsilon} \widehat{\mathbb{A}}_{0} e^{\varphi^{\varepsilon} / \varepsilon}=\varepsilon^{-1} \widehat{a}_{0} \varphi^{\prime}(u)+h_{a}^{\varepsilon}(x) \varphi(u) .
\end{gathered}
$$

Thus, the relation

$$
H^{\varepsilon} \varphi^{\varepsilon}(u, x)=\left[H_{Q}^{\varepsilon}+H_{\varphi}^{\varepsilon}-H_{a}^{\varepsilon}\right] \varphi^{\varepsilon}(u, x)
$$

implies (41) with

$$
\begin{gathered}
\widetilde{\mathbb{A}}(x) \varphi(u):=\widetilde{a}(x) \varphi^{\prime}(u), \\
\widetilde{a}(x):=Q_{0} a(x)-\widehat{a}_{0}
\end{gathered}
$$

(see (24)-25)

Now the solution of the singular perturbation problem [1, Chapter 5] may be used for the equations

$$
\begin{gathered}
Q \varphi_{1}+\widetilde{A}(x) \varphi(u)=0, \quad \Pi \widetilde{A}(x)=0 \\
Q \varphi_{2}-\varphi_{1} Q \varphi_{1}+\frac{1}{2} B_{1}(x)\left[\varphi^{\prime}(u)\right]^{2}=\widehat{H} \varphi(u) .
\end{gathered}
$$

The first equation in (42) has the solution

$$
\varphi_{1}(u, x)=R_{0} \widetilde{a}(x) \varphi^{\prime}(u), \quad Q \varphi_{1}=\widetilde{a}(x) \varphi^{\prime}(u) .
$$

Hence, the second equation in (42) can be rewritten as

$$
Q \varphi_{2}+\frac{1}{2}\left[B_{1}(x)+B_{2}(x)\right]\left[\varphi^{\prime}(u)\right]^{2}=\widehat{H} \varphi(u)
$$

with $B_{2}(x)$ given in (40).

The solvability condition [1, Chapter 5] for the latter equation implies Proposition 4.2.

\section{BIBLIOGRAPHY}

1. V. S. Koroliuk and N. Limnios, Stochastic Systems in Marging Phase Space, VSP, Dordrecht, 2005. MR2205562 (2007a:60004)

2. J. Feng and T. G. Kurtz, Large Deviations for Stochastic Processes, Mathematical Surveys and Monographs, vol. 131, AMS, Providence, RI, 2006. MR2260560 (2009g:60034)

3. A. A. Mogulskii, Large deviations for processes with independent increments, Ann. Probab. 21 (1993), 202-215. MR1207223 (94g:60053)

4. M. J. Freidlin and A. D. Wentzel, Random Perturbation of Dynamical Systems, Springer-Verlag, Berlin, 1998. MR1652127 (99h:60128)

Current address: Institute of Mathematics, Kyiv, Ukraine

E-mail address: korol@imath.kiev.ua

University OF Rome "LA SAPIENZA", ItAly

E-mail address: raimondo.manca@uniroma1.it

Universita'di Chieti, Chieti, Italy

E-mail address: g.damico@unich.it

Received 20/OCT/2012

Originally published in English 